\title{
Clinical and histopathological features of NASH in workers exposed to chemicals with or without associated metabolic conditions
}

\author{
Cotrim HP, De Freitas LAR, Freitas C, Braga L, Sousa R, Carvalho F, \\ Paraná R, Santos-Jesus R, Andrade Z. Clinical and histopathological \\ features of NASH in workers exposed to chemicals with or without \\ associated metabolic conditions. \\ Liver International 2004: 24: 131-135. (C) Blackwell Munksgaard 2004
}

\begin{abstract}
Background/Aims: Non-alcoholic steatohepatitis (NASH) has been associated with exposure to chemicals among workers from an industrial complex in Brazil. We investigated the NASH profile of these individuals associated or not with metabolic conditions. Methods: Eighty-four patients with NASH were classified into three groups: G1, 31 patients exposed to chemicals (benzene, xylene, vinyl chloride and others); G2, 30 exposed patients who also presented with obesity, hyperlipidemia and diabetes; and G3, 23 non-exposed patients who presented with metabolic conditions. Results: G1 and G2 were similar in terms of gender ( $97 \%$ and $100 \%$ males) and age ( $37 \pm 5.4$ and $39 \pm 6.5$ years). In G3, $74 \%$ were males and the age was $48 \pm 3.4$ years $(P<0.05)$. In $\mathrm{G} 2$, obesity was present in $26.6 \%$,

hyperlipidemia in $66.6 \%$ and diabetes in $6.6 \%$. In G3, obesity was observed in $43.4 \%$, hyperlipidemia in $30.4 \%$ and diabetes in $26 \%$. Macro- and microsteatosis were observed in $100 \%$ of cases. Perisinusoidal fibrosis was observed in $71 \%$ patients in $\mathrm{G} 1,80 \%$ in $\mathrm{G} 2$ and $52 \%$ in $\mathrm{G} 3(P<0.05)$. Histological evidence of cholestasis was present in $53 \%$ of cases in $\mathrm{G} 1,50 \%$ in $\mathrm{G} 2$ and $13 \%$ in $\mathrm{G} 3(P<0.05)$. Conclusions: Exposure to chemicals appears to be an independent risk factor for NASH that presents a peculiar profile. It is more frequently seen in men younger than non-exposed ones. Steatosis, fibrosis and cholestasis were frequent histological findings. Co-existing metabolic factors did not seem to influence clinical or histopathological presentation.
\end{abstract}

\section{Helma P. Cotrim, Luiz AR De Freitas, Carolina Freitas, Luciana Braga, Rodrigo Sousa, Fernando Carvalho, Raymundo Paraná, Rogerio Santos- Jesus and Zilton Andrade}

Faculdade de Medicina, Hospital Universitário Prof. Edgar Santos, Universidade Federal da Bahia, Salvador, Brazil
Key words: industrial hepatotoxicity - nonalcoholic steatohepatitis - occupational exposure to chemicals

Helma Pinchemel Cotrim, MD, Avenida Trancredo Neves, Edificio Salvador Trade Center, Torre Norte - Sala 717, CEP: 41830-020 Salvador, Bahia, Brazil.

Tel/fax: 557127562373

e-mail: hpcotrim@ufba.br

Received 16 June 2003,

accepted 6 January 2004
Non-alcoholic steatohepatitis (NASH) is a clinical-pathological condition first described in obese and diabetic women (1-3), which is currently considered to be part of the spectrum of nonalcoholic fatty liver disorders (NAFLDs), ranging from bland steatosis to steatohepatitis and cirrhosis (4-6). NASH has been associated with the metabolic syndrome which includes obesity, diabetes, hypertriglyceridemia and hypertension $(7,8)$. In addition to the usual conditions, NASH has also been associated with jejunal bypass, parenteral nutrition, drugs (corticosteroids, tamoxifen, estrogens) and environmental toxins (9-12).

NASH has been recognized as a frequent liver disease among petrochemical workers from a complex in the Northeast region of Brazil (13,
14). Occupational exposure to chemicals (benzene, xylene, ethylene, dimethylformamide, vinyl chloride and others) was then suspected to be an important risk factor. Improvement in liver enzymes and histology parameters was observed in workers removed from the industrial area (12). However, in these workers occupational exposure appeared either as an independent risk factor or was associated with obesity, hyperlipidemia, type 2 diabetes and so on (metabolic conditions).

This study comparatively describes the clinical and pathological profiles of NASH among patients exposed to chemicals associated or not with metabolic conditions, and consecutive patients with histological NASH who had no exposure to potentially toxic agents. 


\section{Cotrim et al.}

\section{Patients and methods}

Study group selection

The present study involved patients (males and females) with a diagnosis of NASH classified into three groups:

Group 1 (G1): patients for whom exposure to chemicals was the only risk factor for NASH. They had been chronically exposed to several volatile petrochemical products, including benzene, dimethylformamide, ethylene, vinyl chloride and others (see the Appendix) for at least 5 years.

Group 2 (G2): patients whose risk factors for $\mathrm{NASH}$ were exposure to chemicals associated with metabolic conditions (obesity, hyperlipidemia and/or diabetes).

G1 and G2 were initially selected on the basis of having aspartate and alanine aminotransferases (ASTs, ALTs) and/or gamma-glutamyltransferase (GGT) levels at least 1.5 times the upper normal limits in three or more determinations (13).

Group 3 (G3): patients with a diagnosis of NASH associated with metabolic conditions.

G3 was composed of consecutive patients selected from our Liver Unit, who never worked in a petrochemical industry.

\section{Clinical evaluation}

The parameters studied included age, gender and time working in the industrial area, risk factors (obesity, hyperlipidemia, diabetes and exposure to chemicals), liver function and abdominal ultrasound. Obesity was defined as a body mass index (BMI) $\geq 30 \mathrm{~kg} / \mathrm{m}^{2}$. The diagnosis of diabetes was based on the criteria of the American Diabetes Association (15). Aminotransferases were considered to be elevated when their serum levels exceeded 1.5 times the reference level.

The criteria for the diagnosis of NASH were: (a) negative or occasional history of alcohol use ( $<20 \mathrm{~g} /$ day), (b) exclusion of chronic liver disease (viruses $\mathrm{B}$ and $\mathrm{C}$, hemochromatosis and autoimmune diseases), and (c) liver biopsy with a diagnosis of steatohepatitis. Criteria for the diagnosis of NASH related to exposure were: history of exposure, exclusion of others risks factors for NASH (obesity, hyperlipidemia, diabetes, abdominal surgery, drug use, etc.) besides histological and epidemiological data $(12,13,23,24)$.

Histological evaluation

Liver biopsy was performed in all patients. The major indication was persistent elevation of ALT and AST after an attempt to control the metabolic conditions and after 6 or more months of follow-up. Histological classification was according to Matteoni et al. (5). Types 3 (steatosis, ballooning degeneration and inflammation) and 4 (steatosis, ballooning degeneration, inflammation and fibrosis) were considered to be steatohepatitis (NASH). Fibrosis was classified into four stages: stage 0: steatosis without any fibrosis; stage 1: fibrosis limited to the perivenular area in zone 3; stage 2: perivenular and perisinusoidal fibrosis with the formation of thin fibrous septa; stage 3: presence of fibrous septa connecting central venules to central venules and/or central venules to portal tracts. Few parenchymal nodules may be observed; and stage 4: cirrhosis.

Perls' method was used to investigate iron overload.

The Ethics Committee for Medical Research at the University Hospital Edgar Santos - Federal University of Bahia - Brazil approved the study.

\section{Statistical analysis}

Differences among groups were analyzed by oneway ANOVA followed by the Bonferroni test, and all values are reported as mean $\pm \mathrm{SD}$. The data were analyzed using the Epi-info software, version 6.0, and the differences between groups were considered to be significant at $P<0.05$.

\section{Results}

The study was conducted on 84 NASH patients classified into three groups: G1 $(n=31), \mathrm{G} 2$ $(n=30)$ and G3 $(n=23)$. All patients were asymptomatic, including those with cirrhosis detected by histology. Ultrasound was performed in all patients and showed the presence of steatosis. The liver function tests (prothrombin time and albumin levels) were normal, but all subjects presented elevated aminotransferase levels. An ALT/AST ratio greater than 1 was observed in $83.8 \%$ of subjects $(26 / 31)$ in $\mathrm{G} 1$, in $90.0 \%(27 / 30)$ in $\mathrm{G} 2$ and in $74 \%(17 / 23)$ in G3. GGT was elevated in all $(100 \%)$ patients in G1 and G2 and in $69.5 \%$ of subjects $(16 / 23)$ in G3.

Table 1 shows the data for G1, G2 and G3 according to gender, age and time of work (exposure) in the industrial area. Males predominated in all groups. G3 patients with NASH were older than $\mathrm{G} 1$ and $\mathrm{G} 2$ patients $(P=0.002)$. The mean age was $48 \pm 3.4$ years in $G 3,37 \pm 5.4$ years in $\mathrm{G} 1$ and $39 \pm 6.5$ in $\mathrm{G} 2$. The mean time of work in the petrochemical industry was $15 \pm 5.4$ years for both groups (G1 and G2).

Table 2 describes the risk factors for NASH: In $\mathrm{G} 1$, the only risk factor was occupational 
Table 1. Groups of NASH patients according to gender, age and time of work (exposure) in the petrochemical industry

\begin{tabular}{lclll}
\hline & G1 & G2 & G3 & \\
& $n=31$ & $n=30$ & $n=23$ & $P$ \\
\hline Age (years) & $37 \pm 5.4$ & $39 \pm 6.5$ & $48 \pm 3.4$ & 0.002 \\
$\begin{array}{l}\text { Gender } \\
\quad \text { Male }\end{array}$ & $30(97 \%)$ & $30(100 \%)$ & $17(74 \%)$ & \\
$\quad$ Female & $7(3 \%)$ & 0 & $0.6(26 \%)$ & \\
$\begin{array}{l}\text { Time working in the } \\
\quad \text { petrochemical industry } \\
\quad \text { (years) }\end{array}$ & $15 \pm 5.4$ & $15 \pm 4.7$ & - & \\
$\quad$ & & & & \\
\hline
\end{tabular}

NASH, non-alcoholic steatohepatitis; G1, group 1; G2, group 2; G3, group 3.

Table 2. Risk factors associated with NASH in groups 2 and 3

\begin{tabular}{lllll}
\hline Risk factors & G1 & G2 & G3 & \\
& $n=31$ & $n=30$ & $n=23$ & $P$ \\
Exposure & $n(\%)$ & $n(\%)$ & $n(\%)$ & \\
Obesity & $31(100)$ & $30(100)$ & 0 & \\
Hyperlipidemia & 0 & $8(26.6)$ & $10(43.4)$ & \\
Diabetes & 0 & $20(66.6)$ & $7(30.4)$ & 0.01 \\
& 0 & $2(6.0)$ & $6(26)$ & 0.13 \\
\hline
\end{tabular}

$\mathrm{NASH}$, non-alcoholic steatohepatitis; G1, group 1; G2, group 2; G3, group 3.

exposure. In G2, exposure to chemicals was also associated with obesity in $26.6 \%$ (8) of cases, with hyperlipidemia in $66.6 \%$ (20) and with diabetes in $6.6 \%$ (2). In G3, obesity was present in $43.4 \%$ (10) of cases, hyperlipidemia in $30.4 \%$ (17) and diabetes in $26 \%$ (6).

The histological findings in liver biopsies are described in Table 3. All 84 patients presented steatohepatitis (macro- and microsteatosis, inflammation and ballooning degeneration of hepatocytes). Macro-vesicular steatosis was more frequent among exposed subjects and macro- and microvacuolar steatosis was more frequent among non-exposed subjects, but $100 \%$ of patients presented with steatosis. Perisinusoidal fibrosis was observed in $71 \%$ of patients $(22 / 31)$ in $\mathrm{G} 1,80 \%(24 / 30)$ in $\mathrm{G} 2$ and $52 \%(12 / 23)$ in $\mathrm{G} 3$. Two cases of cirrhosis were observed in G3 $(8.6 \%)$. Bile plugs within bile canuliculi were found in $51.6 \%$ of cases in G1, $50 \%$ in G2 and in $13 \%$ of G3. The presence of cholestasis in NASH associated with exposure was statistically significant $(P=0.002)$ and was correlated with elevated serum GGT levels. However, the levels of bilirubin and alkaline phosphatase were normal in $100 \%$ of cases in G1, G2 and G3. Iron overload analyzed by Perls' method was observed in $19.3 \%$ of subjects in G1, $13.3 \%$ in $\mathrm{G} 2$ and $26 \%$ in G3 $(P>0.05)$. Table 4 describes the grades of fibrosis associated with steatohepatitis in liver biopsies. Mild or grade I fibrosis was the most frequent in all groups.
Table 3. Histological findings in liver biopsies from patients with $\mathrm{NASH}$

\begin{tabular}{lllll}
\hline Histological findings & $\begin{array}{l}\mathrm{G} 1 \\
n=31\end{array}$ & $\begin{array}{l}\mathrm{G} 2 \\
n=30\end{array}$ & $\begin{array}{l}\mathrm{G} 3 \\
n=23\end{array}$ & $P$ \\
\hline & $n(\%)$ & $n(\%)$ & $n(\%)$ & \\
$\begin{array}{l}\text { Steatohepatitis without } \\
\quad \text { fibrosis }\end{array}$ & $9(29.0)$ & $6(20.0)$ & $11(47.8)$ & \\
$\begin{array}{l}\text { Steatohepatitis with } \\
\quad \text { fibrosis }\end{array}$ & $22(71)$ & $24(80)$ & $12(52)$ & \\
$\begin{array}{l}\text { Cirrhosis } \\
\text { Cholestasis }\end{array}$ & 0 & 0 & $2(8.6)$ & \\
$\begin{array}{l}\text { Steatohepatitis/iron } \\
\quad \text { overload }\end{array}$ & $6(16(51.6)$ & $12(40)$ & $3(13.0)$ & 0.002 \\
& & $4(13.3)$ & $6(26.0)$ & \\
\end{tabular}

Steatohepatitis: steatosis + ballooning degeneration+inflammation.

$\mathrm{NASH}$, non-alcoholic steatohepatitis; G1, group 1; G2, group 2; G3, group 3.

Table 4. Steatohepatitis and fibrosis in liver biopsies from patients with NASH

\begin{tabular}{lccl}
\hline & G1 & G2 & G3 \\
Fibrosis & $n=31$ & $n=30$ & $n=23$ \\
\hline & $n(\%)$ & $n(\%)$ & $n(\%)$ \\
Grade 0 & $9(29.0)$ & $6(20.0)$ & $11(47.8)$ \\
Grade I (mild) & $19(61.2)$ & $17(56.6)$ & $8(34.7)$ \\
Grade II (moderate) & $3(9.6)$ & $7(23.3)$ & $2(8.6)$ \\
Grade III (severe) & 0 & 0 & 0 \\
Cirrhosis & 0 & 0 & $2(8.6)$
\end{tabular}

NASH, non-alcoholic steatohepatitis; G1, group 1; G2, group 2; G3, group 3.

\section{Discussion}

NASH is considered nowadays to comprise a broad spectrum of conditions involving fatty liver alterations, and is associated with several risk factors and different profiles (16-19). Although NASH has been related to conditions characterized by insulin resistance, it has also been associated with other factors $(7,11)$.

Occupational exposure to chemicals has been associated with steatosis, and steatohepatitis has been described in some studies (11). The solvent dimethylformamide has been related to steatosis and focal hepatocellular necrosis (20). Steatohepatitis was described in painters who work with solvents and thinners (21). In Spain in 1981, NASH was also reported among individuals who developed a multisystem disorder after the consumption of adulterated cooking oil (22).

The spectrum of toxic liver injury related to chemicals is broad, including cholestasis, hepatitis, tumors, thrombosis, steatosis and steatohepatitis. The extent of hepatic damage depends on the dose, duration of exposure, the activity of hepatic drug metabolizing enzymes and individual susceptibility (11). However, the diagnosis of hepatotoxicity and occupational exposure is 


\section{Cotrim et al.}

considered difficult, and evidence is often purely circumstantial $(11,23,24)$.

Clinical and epidemiological studies have related NASH to occupational exposure to chemicals (benzene, xylene, ethylene, dimethylformamide, vinyl chloride and others) among workers from a petrochemical plant in Brazil. After they were removed from the exposed work area, the workers presented a gradual improvement in liver tests and histology (12). The route of exposure was not known. Presumably, the skin and digestive and respiratory tracts were the most likely.

Epidemiological investigations (25) such as prevalence, descriptive and case-control studies on these workers support evidence from these previous studies, showing that occupational exposure is a risk factor for NASH among workers from a petrochemical industry in Brazil (26).

When the spectrum of liver disease was studied in these workers, NASH was the most frequent chronic liver disease and occupational exposure was the condition most frequently associated with it (13). However, the role of metabolic factors in association with exposure in this kind of NASH has not been evaluated before.

The results of the present study show that workers with NASH related to exposure associated or not with metabolic conditions (obesity, hyperlipidemia and type 2 diabetes) were similar in terms of gender, age and biochemical and histological findings. However, patients with NASH exposed to chemicals were younger than non-exposed individuals and the difference was significant. The most frequent conditions associated with exposure in group two (G2 - coexisting metabolic risk) were obesity and hyperlipidemia; however, these concomitant risk factors did not seem to influence the clinical and histological profile of these patients with steatohepatitis. These results suggest that exposure to chemicals is an independent risk factor for NASH in these workers. Preliminary results about the relationship between these petrochemicals and NASH did not reveal insulin resistance by the method of homeostasis assessment. These results could be of help in future studies to better understand NASH related to exposure to chemicals.

Analysis of the histological findings showed that steatohepatitis with fibrosis was frequent among exposed patients and was significant. However, mild fibrosis was predominant in all three groups. Cirrhosis was found in two nonexposed patients.

Ultrastructural study of NASH patients from the petrochemical industry has further detected cholestasis, presence of megamitochondria and increased lipofuchsin deposits. These findings were considered to be suggestive of an underlying hepatotoxic mechanism (12).

In the present study, cholestasis was a significant histological finding among NASH patients from the petrochemical industry and was compatible with elevated GGT levels that were more frequent among exposed patients. Altered GGT was the first parameter observed in workers exposed to chemicals when they were evaluated by periodical exams; however, the levels of bilirubin and alkaline phosphatase were normal.

In conclusion, these results suggest that NASH courses with peculiar features among petrochemical workers. It is most frequent in individuals younger than non-exposed subjects. Concomitant associated metabolic factors did not seem to influence clinical or histological presentation. Occupational exposure may be an independent and most important risk factor for NASH. Fibrosis and cholestasis are frequent histological findings. The long-term prognosis remains uncertain.

\section{Acknowledgements}

The authors thank Stephen H. Caldwell, MD from University of Virginia-USA for his assistance with the preparation of the manuscript.

\section{Appendix}

Petrochemical products most frequently found at the industrial area in this study:

- Acrylonitrile

- Acetylene

- Benzene

- Carbon tetrachloride

- Chloroform

- Chloroethyl vinyl ether

- Dimethylformamide

- Ethane

- Ethylene

- Hexane

- Methanol

- Toluene

- Trinitrotoluene

- Tetrachloroethane

- Vinyl chloride

- Styrene

- Xylenes

- Hydrocarbon mists

\section{References}

1. Adler A, Schaffner F. Fatty liver hepatitis and cirrhosis in obese patients. Am J Med 1979; 67: 811-6.

2. Ludwig J, Viggiano T R, McGill D B, Ott B J. Nonalcoholic steatohepatitis. Mayo Clin Proc 1980; 55: 434-8. 
3. LEE R G. Nonalcoholic steatohepatitis. A study of 49 patients. Hum Pathol 1989; 20: 594-8.

4. Dixon J B, Bhathal P S, O'Brien P E. Nonalcoholic fatty liver disease: predictors of nonalcoholic steatohepatitis and liver fibrosis in the severely obese. Gastroenterology 2001; 121(1): 91-107.

5. Matteoni C A, Younossi Z M, Gramlich T, Boparai N, Liu Y C, McCullough A J. Nonalcoholic fatty liver disease: a spectrum of clinical and pathological severity. Gastroenterology 1999; 116(6): 1413-9.

6. Brunt E M, Janney C J, Di Bisceglie A M, NeuschWANDER-TETRI B A, BACON B R. Non-alcoholic steatohepatitis: a proposal for grading and staging the histologic lesions. Am J Gastroenterol 1999; 94: 2467-74.

7. Marchesini G, Brizi M, Morselli-Labate A M, BianChi G, Bugianesi E, McCullough A J, Forlani G, et al. Association of nonalcoholic fatty liver disease with insulin resistance. Am J Med 1999; 107: 450-5.

8. Chitturi S, Abeygunasekera S, Farrell G C, HolmesWalker J, Hui J M, Fung C, Karim R, et al. NASH and insulin resistance: insulin hypersecretion and specific association with the insulin resistance syndrome. Hepatology 2002; 35: 373-9.

9. Cortez-Pinto H, Camilo M E, Baptista A, De Oliveira A G, De Moura M C. Non-alcoholic fatty liver: another feature of the metabolic syndrome? Clin Nutr 1999; 18(6): $353-8$

10. REID A E. Nonalcoholic steatohepatitis. Gastroenterology 2001; 121: 710-23.

11. Farrell G C. Drugs and steatohepatitis. Semin Liver Dis 2002; 22: 185-94.

12. Cotrim H P, Andrade Z A, Parana R, Portugal M, Lyra L G, Freitas L A. Nonalcoholic steatohepatitis: a toxic liver disease in industrial workers. Liver 1999; 19(4): 299-304.

13. Cotrim H P, Andrade Z A, Parana R, Portugal M, Lyra L G, Freitas L A. Nonalcoholic steatohepatitis (NASH) in Brazil: spectrum from a special population. Hepatology 1999; 30: 136A.

14. Cotrim H P, Andrade Z A, Parana R, Portugal M, Lyra L G, Freitas L A. Importância da esteato-hepatite não alcoólica (NASH) em trabalhadores da indústria petroquímica: GED (Gastroenterologia e Endoscopia Digestiva), 2001; 20: 157-62.

15. Colman P, Yhomas D, Zimmet P, Welborne T, GarciaWebb P, Moore M. New classification and criteria for diagnosis of diabetes mellitus. Med J Aust 1999; 170: $375-8$.

16. Bacon B R, Ffarahyash M I, Janney C G, NeuschwanDER-TETri B A. Nonalcoholic steatohepatitis: an expanded clinical entity. Gastroenterology 1994; 107: 1103-9-1.

17. Ikai E, Ishizaki M, Suzuki Y, Ishida M, Noborizaka Y, YAmada Y. Association between hepatic steatosis, insulin resistance and hyperinsulinemia as related to hypertension in alcohol consumers and obese people. J Hum Hypertens 1995; 9: 101-5.

18. Marchesini G, Brizi M, Bianchi G, Tomassetti S, Bugianesi E, Lenzi M, McCullough AJ, et al. Nonalcoholic fatty liver disease: a feature of the metabolic syndrome. Diabetes 2001; 50: 1844-50.

19. Caldwell S H, Oelsner D H, Iezzoni J C, Hespenheide E E, Battle E H, Driscoll C J. Cryptogenic cirrhosis: clinical characterization and risk factors for underlying disease. Hepatology 1999; 29: 664-9.

20. Redlich C A, West A B, Fleming L, True L D, Cullen M R, Riely C A. Clinical and pathological characteristics of hepatotoxicity associated with occupational exposure to dimethylformamide. Gastroenterology 1990; 99: 748-57.

21. Dossing M, Arlien-Soborg P, Milling Petersen L, RANEK L. Liver damage associated with occupational exposure to organic solvents in house painters. Eur J Clin Invest 1983; 13: 151-7.

22. Solis-Herruzo J Á, Vidal J V, Colina F, Castellano G, Munoz-Yague M T, Morillas J D. Clinico-biochemical evolution and late hepatic lesions in the toxic oil syndrome. Gastroenterology 1987; 93: 558-68.

23. Tolman K G, Sirrine R W. Drug-induced liver disease. Clin Liver Disease 1998; 2(3): 1-31.

24. Dossing M, RaneK L. Isolated liver damage in chemical workers. Br J Int Med 1984; 41: 142-4.

25. Carvalho F M, Silvany-Neto A M, Barberino J L, Gidi J, Guedes F, Oliveira R, Cotrim H P. Epidemiological studies support an occupational etiology for nonalcoholic steatohepatitis among petrochemical workers from Bahia, Brazil. J Hepatol 2001; 34(Suppl. 1): 229-30.

26. Cotrim H P, Carvalho F M, Freitas L A, Parana R, Oliveira C J, Valadares C, Andrade Z. Nonalcoholic steatohepatitis (NASH) and occupational exposure: a casecontrol study among petrochemical workers. J Hepatol 2001; 34(Suppl. 1): 229-30. 